\title{
Barnacles and mussels as biomonitors of trace elements: a comparative study
}

\author{
D. J. H. Phillips ${ }^{1, *}$, P. S. Rainbow ${ }^{2}$ \\ ${ }^{1}$ Environmental Protection Department, Sincere Bldng., Central, Hong Kong \\ ${ }^{2}$ School of Biological Sciences, Queen Mary College, Mile End Rd, London E1 4NS, United Kingdom
}

\begin{abstract}
Concentrations of 5 trace elements (cadmium, chromium, copper, lead, zinc) in 3 species of barnacle and the mussel Perna viridis were determined for up to 18 sites in Hong Kong coastal waters. Although each species accumulated differing absolute amounts of metals, qualitative agreement between contamination profiles exhibited by the 4 species for all elements other than cadmium was excellent. This was the case even for zinc, which is partially regulated by $P$. viridis. The relative bioavailabilities of metals other than cadmium to each of the 4 species at the sites studied are thus similar for the barnacle species and the mussel, and a consistent pattern of environmental contamination emerges from these data. By contrast, the bioavailability of cadmium appears to differ between each species; this may be at least partly due to the lack of a marked gradient in cadmium contamination of Hong Kong waters, as shown by previous studies and confirmed here. The differences between the species in trace metal accumulation are discussed, particularly as they relate to the use of barnacles and mussels as biomonitors of aquatic contamination. It is suggested that these species should be further employed in subtropical and tropical nations to estâblish present levels of contamination and monitor future trends.
\end{abstract}

\section{INTRODUCTION}

The use of aquatic organisms to monitor trace metal abundance and bioavailability in coastal waters is well established, especially in temperate regions (Phillips 1977, 1980, Goldberg et al. 1978, Bryan et al. 1980, 1985). Bivalve molluscs are generally thought to be among the best species for such studies (Phillips 1980), although barnacles have also been used successfully (Rainbow 1987).

One of the advantages of employing biomonitors to assess the abundance of trace metals in aquatic ecosystems is that the element levels found in their tissues are by definition a function of the amounts of bioavailable metals present in the environment. No such measure of bioavailability may be provided by the analysis of contaminants in water or sediments, as too little is known of the relative or absolute availabilities of metals in different forms to biota (Phillips 1980). However, the

\footnotetext{
- Present address: Aquatic Habitat Institute, 180 Richmond Field Station, 1301 South 46th Street, Richmond, California 94804, USA.
}

concept of bioavailability is itself complex, as different organisms may take up metals more or less efficiently from solution, suspension, or food. Studies comparing trace metal profiles from several biomonitors taken at the same locations permit an assessment of the relative bioavailabilities of metals to different species. Unfortunately, such investigations are rare even in temperate regions, and almost nonexistent in warmer waters.

Hong Kong coastal waters are ideal to test the capacity of different organisms to monitor trace metal abundance, as a well defined gradient of contamination exists. Studies of sediments, oysters and mussels have clearly shown trace element enrichment in the heavily urbanized Victoria Harbour area (receiving sewage wastes from 3.7 million inhabitants, and associated industrial effluents), concentrations generally decreasing with distance away from the Harbour (Phillips 1979a, 1985, Phillips \& Yim 1981). The present investigation contrasts contamination profiles found in 4 biomonitor species in these waters, for 5 trace metals (cadmium, chromium, copper, lead, zinc). The species employed were the green-lipped mussel Perna viridis and the barnacles Capitulum mitella. Tetraclita squamosa, and Balanus amphitrite amphitrite. 


\section{MATERIALS AND METHODS}

Mussels and barnacles were sampled from 8 to 16 April, 1986. Sampling locations were based on a previous survey of mussels (Phillips 1985), with a few minor changes or additions to compensate for the differing distributions of the species studied. Mussels Perna viridis (Linnaeus) were taken from 16 locations (Table 1 , Fig. 1). Twenty individuals of shell length 50 to $70 \mathrm{~mm}$ were collected from similar water depths at each site. The 3 barnacle species - Capitulum mitella (Linnaeus), Tetraclita squamosa Bruguière (not distinguished into subspecies $T$. squamosa squamosa Bruguière and $T$. squamosa japonica Pilsbry), and Balanus amphitrite amphitrite Darwin - were collected at all study sites where they were present (Table 1). As C. mitella was found at only 4 of the 16 sites where $P$. viridis was taken, 2 additional collection locations for this barnacle were added (Sites 17 and 18 in Fig. 1 and Table 1 ). Each barnacle species occupied a characteristic zone in the eulittoral or sublittoral, in addition to possessing distinct habitat preferences, especially with respect to wave action (Wu 1973). The effects of size of individuals on trace metal levels in barnacles could not be accounted for by sampling a restricted size range (as for mussels), as many populations consisted predominantly of individuals of a particular size and this differed between locations. The largest individuals present at each site were taken, and statistical treatment of data generated from these was employed (see below) to account for any size effects. For C. mitella and $T$. squamosa, at least 10 individuals were analyzed from each site. The smaller size of $B$. amphitrite amphitrite necessitated pooling of 15 bodies to provide each of 10 replicates at each site.
Table 1. Barnacle species (Balanus amphitrite amphitrite Capitulum mitella and Tetraclita squamosal sampled at each of the 18 locations shown in Fig. 1. Locations 4 to 10 and 17 are in Victoria Harbour, 11 and 12 in Junk Bay, and 13 to 16 in Tolo Harbour and Channel. Perna viridis was sampled at Locations 1 to 16

\begin{tabular}{|cll|}
\hline Code & \multicolumn{1}{c}{ Location } & Barnacle species collected \\
\hline 1 & Tung Chung & $\begin{array}{l}\text { T squamosa, B. amphitrite } \\
\text { amphitrite }\end{array}$ \\
2 & Reef Island & C. mitella; $T$. squamosa \\
3 & Chai Wan Kok & B. amphitrite amphitrite \\
4 & Kennedy Town & None \\
5 & Queens Pier & C. mitella; $T$ squamosa \\
6 & Kowloon Pier & T squamosa \\
7 & Hung Hom & C. mitella; $T$ squamosa \\
8 & Causeway Bay & C. mitella; T. squamosa \\
9 & North Point & T. squamosa; B. amphitrite \\
& & amphitrite \\
10 & Kwun Tong & B. amphitrite amphitrite \\
11 & Rennies Mill & B. amphitrite amphitrite \\
12 & Hang Hau & B. amphitrite amphitrite \\
13 & Sha Tin & B. amphitrite amphitrite \\
14 & Tai Po Kau & B. amphitrite amphitrite \\
15 & Wu Kwai Sha & B. amphitrite amphitrite \\
16 & Lai Chi Chong & B. amphitrite amphitrite \\
17 & Stonecutters Island & C. mitella \\
18 & Cape D'Aguilar & C. mitella \\
\end{tabular}

All species were kept cool during transport to the laboratory. No depuration period was employed, as this may lead to contamination of samples. In any event, depuration has little effect on trace metal levels in the species studied (NAS 1980, Latouche \& Mix 1982, Phillips 1985, Rainbow unpubl.).

Mussel samples were stored frozen at $-20^{\circ} \mathrm{C}$ until required for analysis. Upon thawing, the whole soft parts were removed using stainless steel instruments
Fig. 1. Hong Kong coastal waters, showing 18 locations at which samples of barnacles (Capitulum mitella, Tetraclita squamosa, Balanus amphitrite amphitrite) and/or mussels (Perna viridis) were taken. Locations 4 to 10 and 17 are in Victoria Harbour; 11 and 12, in Junk Bay; and 13 to 16, in. Tolo Harbour and Channel. Inset: Hong Kong in relation to southern China

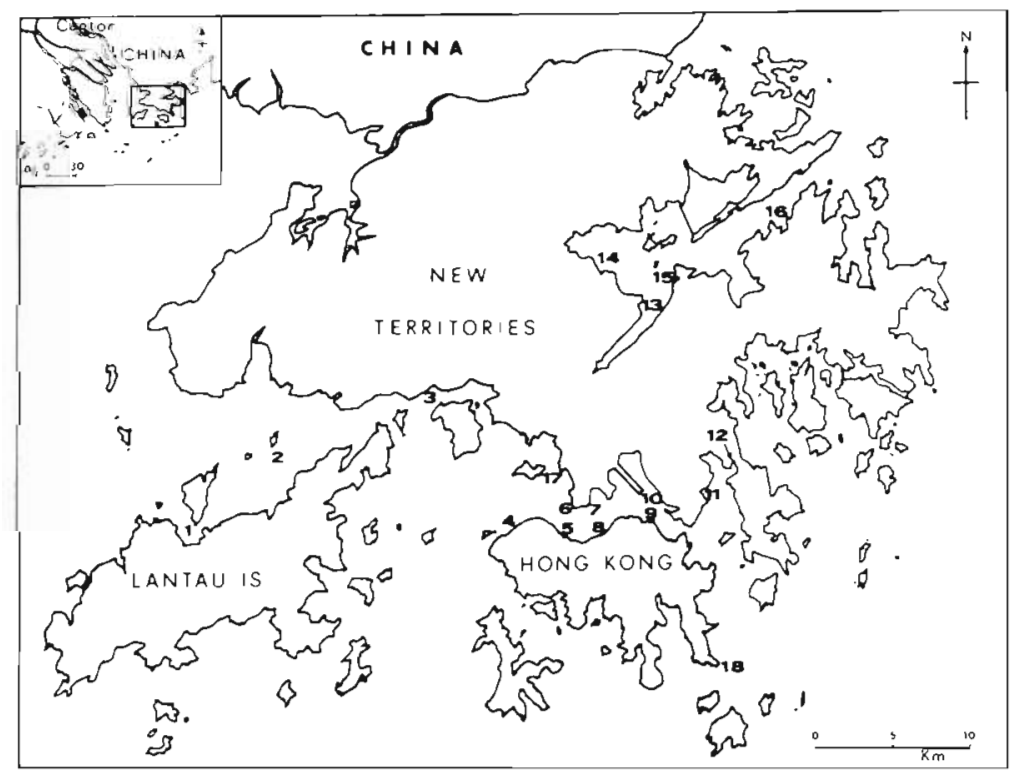


(byssus was discarded). Mussels were analyzed in 5 replicates of 4 individuals for each site, and were homogenized using a Brinkman PT 10-35 Polytron Kinematica ultrasonic blender (confirmed to be noncontaminating in previous studies). Aliquots of $10 \mathrm{~g}$ wet weight were digested with $20 \mathrm{ml}$ concentrated $\mathrm{HNO}_{3}$ at $120^{\circ} \mathrm{C}$ and made up to $100 \mathrm{ml}$ with distilled deionized water for analysis. Separate aliquots were dried at $100^{\circ} \mathrm{C}$ to constant weight to define wet weight:dry weight ratios. Copper and zinc were analyzed by flame atomic absorption spectrophotometry (AAS) using a Hitachi 180-80 Zeeman spectrophotometer. The other elements were quantified by graphite furnace AAS, on either a Perkin-Elmer Zeeman/3030 instrument (chromium, lead) or a Hitachi 180-80 Polarized Zeeman instrument (cadmium). Barnacle samples, consisting of individual or pooled bodies, were dried in acid-washed plastic vials to constant weight, prior to digestion at $100^{\circ} \mathrm{C}$ in concentrated $\mathrm{HNO}_{3}$. Digests were typically made up to $5 \mathrm{ml}$ with double-distilled water. Analysis was by flame AAS on either a Varian AA 375 or an IL 157 spectrophotometer. Throughout all analyses, quality control was provided by concurrent analytical checks on certified reference materials. These included NIES material no. 6 (mussel) for work on Perna viridis, and NRC Canada TORT-1 (lobster hepatopancreas) for barnacle analyses. Agreement with certified values was good throughout.

Statistical data treatment employed 2 parametric techniques. Pooling of tissue from mussels of similar sizes from each site overcame possible effects of mussel size on metal concentrations. It is possible, therefore, to compare mean metal concentrations in mussel soft tissue from each site by analysis of variance (ANOVA). In the case of each metal, sample variances increased with means and it was necessary to use logarithmically-transformed values to ensure normal distributions of data. For barnacles, it was first necessary to check whether any residual effects of body size on metal concentrations remained after choice of the largest available specimens at each site and after any pooling of bodies (for Balanus amphitrite amphitrite). Data for each metal at each site were checked for significant correlations and regressions between metal concentration $(y)$ in $\mu \mathrm{g} \mathrm{g}^{-1}$ dry weight and body dry weight $(x)$ in $g$ (or mean body dry weight in the case of pooled bodies). In many cases, significant regressions $(p<0.05)$ were found, the data often being a better fit to a straight line after logarithmic transformation [log metal concentration ( $y$ ) against log dry weight $(x)]$. Transforming the data to log values also has the advantage of further normalizing the data, a prerequisite for the use of parametric statistics.

To allow for size effects, analysis of covariance (ANCOVA) was employed. ANCOVA tests for signifi- cant differences in values of $y$ (log metal concentration) between regression lines fitted to each set of data, having allowed for differences in values of $x$ (log dry weight). In effect, ANCOVA compares predicted metal concentrations of bodies of a particular barnacle species from several sites at a standardized body dry weight. The weights chosen represented approximate medians of the ranges of body weights for each species of barnacle; these were $0.05 \mathrm{~g}$ for Capitulum mitella, $0.02 \mathrm{~g}$ for Tetraclita squamosa, and $0.004 \mathrm{~g}$ for Balanus amphitrite amphitrite.

ANOVA and ANCOVA treatments thus ranked analytical data for each element and species in decreasing order of contamination of samples. Comparisons between the ranking of sites for each metal in mussels and barnacles employed Spearman's rank correlation.

\section{RESULTS}

Results for each metal are presented graphically and in Tables 2 to 6 . Data for barnacles are shown as estimated concentrations in barnacle bodies of standardized weight, generated from ANCOVA analyses, with $95 \%$ confidence limits. Concentrations of metals are assigned letters (A, B, C, etc.) in tables from ANCOVA (barnacle) or ANOVA (mussel) data; these differ where significantly different element concentrations were present in different samples. Sites are ranked in approximate order of decreasing concentration of trace elements. In Figs. 2 to 6 , such ranking of metal levels is shown by the use of circles of differing diameters.

Cadmium concentrations in mussels and barnacles are shown in Table 2 and Fig. 2. Each species exhibited somewhat elevated levels of cadmium at Reef Island, but differences in contamination profiles existed for other sites between species. Barnacle data suggest relatively high cadmium bioavailability at North Point and in Victoria Harbour, whereas Perna viridis attained highest cadmium levels at Rennies Mill. Comparison of rank orders of sites from barnacle and mussel data using Spearman's rank correlation tests revealed no significant correlation $\left(r_{s}=0.514, n=15, N S\right)$, even if data from Rennies Mill were excluded $\left(\mathrm{r}_{\mathrm{s}}=0.495, n=\right.$ 14, NS). This suggests that barnacles and $P$. viridis differ with respect to their net uptake of cadmium, i.e. that cadmium bioavailability to barnacles is distinct from that to green-lipped mussels. It is also notable that little variation in cadmium concentrations between sites was evident, confirming earlier conclusions (Phillips 1979a, 1985) that no major cadmium source or contamination gradient exists in Hong Kong waters

Concentrations of chromium in the species analyzed are presented in Table 3 and Fig. 3. No data are 
Table 2. Concentrations of cadmium ( $\mu \mathrm{g} \mathrm{g}^{-1}$ dry wt) and respective confidence limits (CL) in barnacles and mussels from Hong Kong waters, ranked in approximate order of decreasing contamination of samples. Letters in ANCOVA and ANOVA columns denote presence or absence or significant differences between samples

\begin{tabular}{|c|c|c|c|c|c|c|c|c|c|c|c|c|}
\hline \multirow[t]{2}{*}{ Location (code) } & \multicolumn{3}{|c|}{ Capitulum mitella } & \multicolumn{3}{|c|}{ Tetraclita squamosa } & \multicolumn{3}{|c|}{$\begin{array}{c}\text { Balanus amphitrite } \\
\text { amphitrite }\end{array}$} & \multicolumn{3}{|c|}{ Perna viridis } \\
\hline & $(\mathrm{Cd})$ & CL & ANCOVA & $(\mathrm{Cd})$ & $\mathrm{CL}$ & ANCOVA & $(\mathrm{Cd})$ & $\mathrm{CL}$ & ANCOVA & $(\mathrm{Cd})$ & CL & ANOVA \\
\hline Reef Island (2) & 4.4 & $\begin{array}{l}5.7 \\
3.4\end{array}$ & B & 7.7 & $\begin{array}{r}12.6 \\
4.7\end{array}$ & A & & & & 1.06 & $\begin{array}{l}1.31 \\
0.81\end{array}$ & B \\
\hline North Point (9) & & & & 6.1 & $\begin{array}{l}7.9 \\
4.7\end{array}$ & A & 10.1 & $\begin{array}{r}11.5 \\
8.9\end{array}$ & A & 0.22 & $\begin{array}{l}0.36 \\
0.08\end{array}$ & $E, F$ \\
\hline Causeway Bay (8) & 5.2 & $\begin{array}{l}6.9 \\
4.0\end{array}$ & A & 5.4 & $\begin{array}{r}20.0 \\
1.4\end{array}$ & A & & & & 0.36 & $\begin{array}{l}0.53 \\
0.19\end{array}$ & $\mathrm{D}$ \\
\hline Chai Wan Kok (3) & & & & & & & 7.3 & $\begin{array}{l}8.9 \\
5.9\end{array}$ & B & 0.51 & $\begin{array}{l}0.93 \\
0.09\end{array}$ & $\mathrm{C}$ \\
\hline Kwun Tong (10) & & & & & & & 6.9 & $\begin{array}{l}8.8 \\
5.5\end{array}$ & B & 0.52 & $\begin{array}{l}0.69 \\
0.35\end{array}$ & $\mathrm{C}$ \\
\hline Hung Hom (7) & 10.0 & $\begin{array}{r}15.9 \\
5.2\end{array}$ & A & 2.8 & $\begin{array}{l}5.2 \\
1.5\end{array}$ & $B$ & & & & 0.34 & $\begin{array}{l}0.45 \\
0.23\end{array}$ & $\mathrm{D}$ \\
\hline Stonecutters (17) & 7.2 & $\begin{array}{r}10.2 \\
5.0\end{array}$ & A & & & & & & & & & \\
\hline Rennies Mill (11) & & & & & & & 5.8 & $\begin{array}{l}7.3 \\
4.7\end{array}$ & C & 1.48 & $\begin{array}{l}2.67 \\
0.29\end{array}$ & A \\
\hline Tung Chung (1) & & & & 4.2 & $\begin{array}{l}6.5 \\
2.7\end{array}$ & B & 2.7 & $\begin{array}{c}7 \times 10^{7} \\
0\end{array}$ & $\mathrm{C}$ & 0.52 & $\begin{array}{l}0.63 \\
0.41\end{array}$ & $\mathrm{C}$ \\
\hline Kennedy Town (4) & & & & & & & & & & 0.44 & $\begin{array}{l}0.66 \\
0.22\end{array}$ & $\mathrm{C}$ \\
\hline Hang Hau (12) & & & & & & & 4.2 & $\begin{array}{l}5.1 \\
3.4\end{array}$ & C & 0.39 & $\begin{array}{l}0.58 \\
0.20\end{array}$ & C \\
\hline Kowloon Pier (6) & & & & 3.8 & $\begin{array}{l}4.9 \\
3.0\end{array}$ & B & & & & 0.27 & $\begin{array}{l}0.41 \\
0.13\end{array}$ & $\mathrm{E}$ \\
\hline Cape D'Aguilar (18) & 5.2 & $\begin{array}{l}5.7 \\
4.7\end{array}$ & B & & & & & & & & & \\
\hline Queens Pier (5) & 2.9 & $\begin{array}{l}4.4 \\
1.9\end{array}$ & B & 3.6 & $\begin{array}{l}4.4 \\
2.9\end{array}$ & $\mathrm{~B}$ & & & & 0.34 & $\begin{array}{l}0.51 \\
0.17\end{array}$ & $\mathrm{D}$ \\
\hline Wu Kwai Sha (15) & & & & & & & 4.4 & $\begin{array}{l}5.0 \\
3.9\end{array}$ & $\mathrm{C}$ & 0.20 & $\begin{array}{l}0.26 \\
0.14\end{array}$ & F \\
\hline Lai Chi Chong (16) & & & & & & & 5.5 & $\begin{array}{r}22.6 \\
1.4\end{array}$ & $\mathrm{C}$ & 0.19 & $\begin{array}{l}0.30 \\
0.08\end{array}$ & F \\
\hline Tai Po Kau (14) & & & & & & & 4.1 & $\begin{array}{l}7.4 \\
2.2\end{array}$ & $\mathrm{C}$ & 0.15 & $\begin{array}{l}0.29 \\
0.01\end{array}$ & F \\
\hline Sha Tin (13) & & & & & & & 2.1 & $\begin{array}{l}2.5 \\
1.7\end{array}$ & D & 0.20 & $\begin{array}{l}0.23 \\
0.17\end{array}$ & F \\
\hline
\end{tabular}

available for Tetraclita squamosa, as chromium levels were too low to permit reliable quantification in this species. Data for the other 2 barnacle species and for mussels exhibited a high correlation between the ranking of sites $\left(\mathrm{r}_{\mathrm{s}}=0.891, n=15, p<0.001\right)$, indicating excellent agreement between chromium contamination profiles. This implies that the bioavailability of chromium to each species analyzed is similar. Concentrations of chromium were highest in all species at Chai Wan Kok, and elevated levels were also found at Kwun Tong and in the remainder of Victoria Harbour.

Data for copper are shown in Table 4 and Fig. 4. As seen for chromium, barnacle and mussel data present a consistent pattern of contamination. Samples from Chai Wan Kok and Kwun Tong exhibited notably elevated levels of copper, and the Victoria Harbour area was again generally contaminated. Comparison of site ranking shows a highly significant correlation between barnacles and mussels $\left(\mathrm{r}_{\mathrm{s}}=0.902, n=15, p<0.001\right)$.

Concentrations of lead in the species analyzed are presented in Table 5 and Fig. 5. The contamination profile for lead was distinct from that for other elements, highest concentrations being found in sites in Junk Bay (Hang Hau and Rennies Mill), with mussels from Chai Wan Kok and Kennedy Town also exhibiting heavy contamination. Lead levels in samples from Vic- 
Table 3. Concentrations of chromium ( $\mu \mathrm{g} \mathrm{g}^{-1} \mathrm{dry}$ wt) and respective confidence limits (CL) in barnacles and mussels from Hong Kong waters, ranked in approximate order of decreasing contamination of samples. Letters in ANCOVA and ANOVA columns denote presence or absence of significant differences between samples

\begin{tabular}{|c|c|c|c|c|c|c|c|c|c|}
\hline \multirow{2}{*}{ Location (code) } & \multicolumn{3}{|c|}{ Capitulum mitella } & \multicolumn{3}{|c|}{ Balanus amphitrite amphitrite } & \multicolumn{3}{|c|}{ Perna viridis } \\
\hline & $(\mathrm{Cr})$ & $\mathrm{CL}$ & ANCOVA & $(\mathrm{Cr})$ & $\mathrm{CL}$ & ANCOVA & $(\mathrm{Cr})$ & $\mathrm{CL}$ & ANOVA \\
\hline \multirow[t]{2}{*}{ Chai Wan Kok (3) } & & & & 28.0 & 35.0 & $A$ & 37.6 & 44.8 & A \\
\hline & & & & & 22.4 & & & 30.4 & \\
\hline \multirow[t]{2}{*}{ Kwun Tong (10) } & & & & 12.9 & 14.8 & B & 16.9 & 25.9 & B \\
\hline & & & & & 11.2 & & & 7.9 & \\
\hline \multirow[t]{2}{*}{ Hung Hom (7) } & 48.5 & 355 & A & & & & 7.6 & 9.1 & $\mathrm{C}$ \\
\hline & & 6.6 & & & & & & 6.1 & \\
\hline \multirow[t]{2}{*}{ Stonecutters (17) } & 7.8 & 16.4 & A & & & & & & \\
\hline & & 5.7 & & & & & & & \\
\hline \multirow[t]{2}{*}{ Queens Pier (5) } & 6.3 & 9.2 & B & & & & 12.5 & 35.5 & $B$ \\
\hline & & 4.3 & & & & & & 0 & \\
\hline \multirow[t]{2}{*}{ Kennedy Town (4) } & & & & & & & 9.5 & 14.5 & B \\
\hline & & & & & & & & 4.5 & \\
\hline \multirow{2}{*}{ Causeway Bay (8) } & 7.4 & 9.7 & B & & & & 6.6 & 12.5 & $\mathrm{C}$ \\
\hline & & 5.6 & & & & & & 0.7 & \\
\hline \multirow[t]{2}{*}{ Kowloon Pier (6) } & & & & & & & 5.2 & 8.3 & $\mathrm{D}$ \\
\hline & & & & & & & & 2.1 & \\
\hline \multirow[t]{2}{*}{ Reef Island (2) } & 3.3 & 5.2 & $\mathrm{C}$ & & & & 4.7 & 9.1 & $\mathrm{D}$ \\
\hline & & 2.1 & & & & & & 0.3 & \\
\hline \multirow[t]{2}{*}{ North Point (9) } & & & & 5.0 & 6.1 & C & 4.5 & 7.9 & $\mathrm{D}$ \\
\hline & & & & & 4.0 & & & 1.1 & \\
\hline \multirow[t]{2}{*}{ Rennies Mill (11) } & & & & 3.7 & 5.8 & $\mathrm{C}$ & 4.1 & 5.5 & $\mathrm{D}$ \\
\hline & & & & & 2.4 & & & 2.7 & \\
\hline \multirow[t]{2}{*}{ Sha Tin (13) } & & & & 3.4 & 4.1 & $\mathrm{D}$ & 3.7 & 5.7 & $\mathrm{D}$ \\
\hline & & & & & 2.8 & & & 1.7 & \\
\hline \multirow[t]{2}{*}{ Tung Chung (1) } & & & & & & & 3.0 & 8.0 & $\mathrm{D}$ \\
\hline & & & & & & & & 0 & \\
\hline \multirow[t]{2}{*}{ Hang Hau (12) } & & & & 3.0 & 4.0 & $\mathrm{D}$ & 3.0 & 6.1 & $\mathrm{D}$ \\
\hline & & & & & 2.3 & & & 0 & \\
\hline \multirow[t]{2}{*}{ Wu Kwai Sha (15) } & & & & 1.8 & 2.3 & $\mathrm{E}$ & 5.1 & 12.1 & $\mathrm{D}$ \\
\hline & & & & & 1.4 & & & 0 & \\
\hline \multirow[t]{2}{*}{ Tai Po Kau (14) } & & & & 0.22 & $6 \times 10^{8}$ & $\mathrm{~F}$ & 3.0 & 7.6 & $\mathrm{D}$ \\
\hline & & & & & 0 & & & 0 & \\
\hline \multirow[t]{2}{*}{ Lai Chi Chong (16) } & & & & 0.55 & 1.7 & $\mathrm{~F}$ & 1.2 & 2.1 & $E$ \\
\hline & & & & & 0.18 & & & 0.3 & \\
\hline \multirow[t]{2}{*}{ Cape D'Aguilar (18) } & 0.98 & 6.6 & $\mathrm{D}$ & & & & & & \\
\hline & & 0.15 & & & & & & & \\
\hline
\end{tabular}

toria Harbour were generally greater than those from north-eastern waters. Comparison of the ranking of sites in barnacle and mussel data again reveals a highly significant correlation $\left(\mathrm{r}_{\mathrm{s}}=0.890, n=15, p<0.001\right)$.

Data for zinc are shown in Table 6 and Fig. 6. All 3 barnacle species exhibited a wide range in accumulated zinc concentrations, with considerable site-to-site variation which was generally consistent between species. This implies the existence of large variability in bioavailable zinc concentrations between sites, as suggested by previous studies of oysters and sediments from these waters (Phillips 1979a, Phillips \& Yim 1981). By contrast, zinc concentrations in Perna viridis varied over a much restricted range, with means for different sites ranging only from 55 to $153 \mu \mathrm{g} \mathrm{g}^{-1}$ dry weight. These data agree well with the previous results of Phillips (1985) for $P$. viridis and suggest that this mussel partially regulates its tissue zinc concentrations. Notwithstanding such differences in absolute variability between zinc levels in the 4 species analyzed, the rank orders of sites from barnacle and mussel data exhibit a highly significant correlation $\left(\mathrm{r}_{\mathrm{s}}=0.856, n=15\right.$, $p<0.001$ ). Sites in Victoria Harbour and Junk Bay are of greatest zinc bioavailability, as noted in previous studies of different biomonitor species and sediments (Phillips 1979a, Phillips \& Yim 1981).

\section{DISCUSSION AND CONCLUSIONS}

The fact that the analysis of biomonitors for contaminants provides a direct measure of pollutant bioavailability in aquatic ecosystems (Phillips 1980) has been a major driving force in the trend towards the use 
Table 4. Concentrations of copper ( $\mu \mathrm{g} \mathrm{g}^{-1}$ dry wt) and respective confidence limits (CL) in barnacles and mussels from Hong Kong waters, ranked in approximate order of decreasing contamination of samples. Letters in ANCOVA and ANOVA columns denote presence or absence of significant differences between samples

\begin{tabular}{|c|c|c|c|c|c|c|c|c|c|c|c|c|}
\hline \multirow[t]{2}{*}{ Location (code) } & \multicolumn{3}{|c|}{ Capitulum mitella } & \multicolumn{3}{|c|}{ Tetraclita squamosa } & \multicolumn{3}{|c|}{$\begin{array}{c}\text { Balanus amphitrite } \\
\text { amphitrite }\end{array}$} & \multicolumn{3}{|c|}{ Perna viridis } \\
\hline & $(\mathrm{Cu})$ & $\mathrm{CL}$ & ANCOVA & $(\mathrm{Cu})$ & $\mathrm{CL}$ & ANCOVA & $(\mathrm{Cu})$ & $\mathrm{CL}$ & ANCOVA & $(\mathrm{Cu})$ & $C L$ & ANOVA \\
\hline Chai Wan Kok (3) & & & & & & & 3472 & $\begin{array}{l}3950 \\
3052\end{array}$ & A & 219 & $\begin{array}{l}338 \\
100\end{array}$ & A \\
\hline Kwun Tong (10) & & & & & & & 2574 & $\begin{array}{l}3414 \\
1940\end{array}$ & B & 149 & $\begin{array}{r}209 \\
88.6\end{array}$ & B \\
\hline North Point (9) & & & & 203 & $\begin{array}{l}243 \\
170\end{array}$ & A & 1010 & $\begin{array}{r}1387 \\
869\end{array}$ & C & 26.7 & $\begin{array}{r}44.9 \\
8.5\end{array}$ & $F$ \\
\hline Hung Hom (7) & 545 & $\begin{array}{r}1290 \\
375\end{array}$ & A & 94.9 & $\begin{array}{r}129 \\
69.9\end{array}$ & B & & & & 60.1 & $\begin{array}{l}81.6 \\
38.6\end{array}$ & $\mathrm{C}$ \\
\hline Kowloon Pier (6) & & & & 80.7 & $\begin{array}{l}92.0 \\
70.7\end{array}$ & B & & & & 24.7 & $\begin{array}{l}34.5 \\
14.9\end{array}$ & F \\
\hline Stonecutters (17) & 537 & $\begin{array}{r}1170 \\
247\end{array}$ & A & & & & & & & & & \\
\hline Queens Pier (5) & 154 & $\begin{array}{r}435 \\
54.4\end{array}$ & B & 80.1 & $\begin{array}{l}91.3 \\
70.3\end{array}$ & B & & & & 24.0 & $\begin{array}{l}30.2 \\
17.8\end{array}$ & $F$ \\
\hline Causeway Bay (8) & 132 & $\begin{array}{r}232 \\
74.6\end{array}$ & $\mathrm{~B}$ & 69.2 & $\begin{array}{l}98.2 \\
48.7\end{array}$ & B & & & & 38.5 & $\begin{array}{l}52.2 \\
24.8\end{array}$ & $D$ \\
\hline Kennedy Town (4) & & & & & & & & & & 29.1 & $\begin{array}{l}38.6 \\
19.6\end{array}$ & E \\
\hline Hang Hau (12) & & & & & & & 486 & $\begin{array}{l}639 \\
373\end{array}$ & $\mathrm{D}$ & 20.8 & $\begin{array}{r}36.5 \\
5.1\end{array}$ & $\mathrm{~F}$ \\
\hline Reef Island (2) & 36.5 & $\begin{array}{l}53.4 \\
25.0\end{array}$ & $\mathrm{C}$ & 30.9 & $\begin{array}{l}41.3 \\
23.2\end{array}$ & C & & & & 20.8 & $\begin{array}{r}34.6 \\
7.0\end{array}$ & $F$ \\
\hline Rennies Mill (11) & & & & & & & 304 & $\begin{array}{l}368 \\
251\end{array}$ & $E$ & 13.5 & $\begin{array}{r}18.9 \\
8.1\end{array}$ & G \\
\hline Tung Chung (1) & & & & 14.9 & $\begin{array}{r}24.3 \\
9.1\end{array}$ & $\mathrm{D}$ & 295 & $\begin{array}{r}13670 \\
6.4\end{array}$ & E & 9.5 & $\begin{array}{r}13.3 \\
5.7\end{array}$ & $\mathrm{H}$ \\
\hline Wu Kwai Sha (15) & & & & & & & 213 & $\begin{array}{l}244 \\
187\end{array}$ & $\mathrm{~F}$ & 15.9 & $\begin{array}{r}24.8 \\
7.0\end{array}$ & $G$ \\
\hline Tai Po Kau (14) & & & & & & & 142 & $\begin{array}{r}235 \\
86.2\end{array}$ & G & 11.2 & $\begin{array}{r}19.0 \\
3.4\end{array}$ & G \\
\hline Sha Tin (13) & & & & & & & 116 & $\begin{array}{r}136 \\
98.5\end{array}$ & $\mathrm{H}$ & 12.6 & $\begin{array}{r}15.3 \\
9.9\end{array}$ & $G$ \\
\hline Lai Chi Chong (16) & & & & & & & 59.3 & $\begin{array}{r}195 \\
18.0\end{array}$ & $\mathrm{H}$ & 10.9 & $\begin{array}{r}14.8 \\
7.0\end{array}$ & $\mathrm{H}$ \\
\hline Cape D'Aguilar (18) & 29.2 & $\begin{array}{l}35.6 \\
24.1\end{array}$ & $\mathrm{D}$ & & & & & & & & & \\
\hline
\end{tabular}

of organisms to monitor water quality. However, it is clear that bioavailability is not of an absolute nature, as some organisms are able to accumulate contaminants that other species do not take up. This is likely to depend largely upon general feeding habits and specific dietary preferences. Thus, for example, deposit feeders may respond to metals in different portions or phases of the biosphere to organisms feeding on phytoplankton. Similarly, differences between contaminant levels in various species at the base of the food chain may translate to divergent rates of accumulation of such toxicants by higher organisms, if specific dietary preferences exist.

As a result of such differences, it should not be surprising that contamination profiles produced by the study of several species do not always agree qualita- tively or quantitatively. Bryan \& Hummerstone (1977) found that the pattern of silver accumulation in several organisms from the Looe Estuary, UK, varied between species. Macroalgae and the herbivorous limpet Patella vulgata exhibited little variation in silver concentrations with distance upriver, while filter-feeders such as the cockle Cerastoderma edule exhibited elevated silver levels in upriver locations. Such differences were also reported for contaminant profiles in macroalgae (Fucus vesiculosus) and mussels (Mytilus edulis) from the Sound between Denmark and Sweden by Phillips (1979b). In these cases, divergent contamination profiles were found in the species studied because the ratio of toxicant levels in solution to those in suspension or food varied non-systematically between the study sites. The importance of dietary habits was emphasized by 
Table 5. Concentrations of lead ( $\mu \mathrm{g} \mathrm{g}^{-1}$ dry wt) and respective confidence limits (CL) in barnacles and mussels from Hong Kong waters, ranked in approximate order of decreasing contamination of samples. Letters in ANCOVA and ANOVA columns denote presence or absence of significant differences between samples

\begin{tabular}{|c|c|c|c|c|c|c|c|c|c|c|c|c|}
\hline \multirow[t]{2}{*}{ Location (code) } & \multicolumn{3}{|c|}{ Capitulum mitella } & \multicolumn{3}{|c|}{ Tetraclita squamosa } & \multicolumn{3}{|c|}{$\begin{array}{c}\text { Balanus amphitrite } \\
\text { amphitrite }\end{array}$} & \multicolumn{3}{|c|}{ Perna viridis } \\
\hline & $(\mathrm{Pb})$ & $\mathrm{CL}$ & ANCOVA & $(\mathrm{Pb})$ & $\mathrm{CL}$ & ANCOVA & $(\mathrm{Pb})$ & $\mathrm{CL}$ & ANCOVA & $(\mathrm{Pb})$ & CL & ANOVA \\
\hline Hang Hau (12) & & & & & & & 39.2 & $\begin{array}{l}68.6 \\
22.4\end{array}$ & A & 40.8 & $\begin{array}{l}50.0 \\
31.6\end{array}$ & A \\
\hline Rennies Mill (11) & & & & & & & 36.5 & $\begin{array}{l}45.2 \\
29.4\end{array}$ & A & 45.6 & $\begin{array}{l}79.7 \\
11.5\end{array}$ & A \\
\hline Chai Wan Kok (3) & & & & & & & 12.7 & $\begin{array}{l}16.0 \\
10.0\end{array}$ & $\mathrm{~B}$ & 47.8 & $\begin{array}{l}109 \\
0\end{array}$ & A \\
\hline Kennedy Town (4) & & & & & & & & & & 37.5 & $\begin{array}{l}62.2 \\
12.8\end{array}$ & A \\
\hline Hung Hom (7) & 8.5 & $\begin{array}{r}14.1 \\
5.1\end{array}$ & A & 4.4 & $\begin{array}{l}6.9 \\
2.7\end{array}$ & A & & & & 14.9 & $\begin{array}{r}19.9 \\
9.9\end{array}$ & B \\
\hline Stonecutters (17) & 6.6 & $\begin{array}{r}13.5 \\
3.2\end{array}$ & A & & & & & & & & & \\
\hline Kowloon Pier (6) & & & & 4.3 & $\begin{array}{r}31.2 \\
0.6\end{array}$ & A & & & & 11.7 & $\begin{array}{r}22.5 \\
0.9\end{array}$ & B \\
\hline Causeway Bay (8) & 4.3 & $\begin{array}{l}6.8 \\
2.7\end{array}$ & A & & & & & & & 10.2 & $\begin{array}{r}18.8 \\
1.6\end{array}$ & B \\
\hline Queens Pier (5) & 3.1 & $\begin{array}{l}5.9 \\
1.6\end{array}$ & B & 3.9 & $\begin{array}{l}5.7 \\
2.7\end{array}$ & A & & & & 12.7 & $\begin{array}{r}23.2 \\
2.2\end{array}$ & B \\
\hline North Point (9) & & & & 3.9 & $\begin{array}{l}5.1 \\
3.0\end{array}$ & A & 8.5 & $\begin{array}{r}13.8 \\
5.2\end{array}$ & C & 10.5 & $\begin{array}{r}18.6 \\
2.4\end{array}$ & B \\
\hline Reef Island (2) & 4.1 & $\begin{array}{r}13.1 \\
1.3\end{array}$ & B & 4.4 & $\begin{array}{r}10.6 \\
1.8\end{array}$ & A & & & & 5.3 & $\begin{array}{l}8.4 \\
2.2\end{array}$ & $\mathrm{C}$ \\
\hline Cape D'Aguilar (18) & 3.0 & $\begin{array}{l}3.4 \\
2.6\end{array}$ & B & & & & & & & & & \\
\hline Tung Chung (1) & & & & 2.2 & $\begin{array}{l}5.1 \\
0.9\end{array}$ & B & 12.7 & $\begin{array}{c}3 \times 10^{11} \\
0\end{array}$ & $B$ & 4.1 & $\begin{array}{l}6.6 \\
1.6\end{array}$ & $\mathrm{C}$ \\
\hline Kwun Tong (10) & & & & & & & 4.1 & $\begin{array}{l}8.1 \\
2.0\end{array}$ & $\mathrm{C}$ & 11.1 & $\begin{array}{r}16.9 \\
5.3\end{array}$ & B \\
\hline Wu Kwai Sha (15) & & & & & & & 9.2 & $\begin{array}{r}12.1 \\
7.0\end{array}$ & $\mathrm{C}$ & 5.7 & $\begin{array}{l}8.5 \\
2.9\end{array}$ & $\mathrm{C}$ \\
\hline Tai Po Kau (14) & & & & & & & 3.8 & $\begin{array}{l}8.3 \\
1.7\end{array}$ & $\mathrm{C}$ & 3.7 & $\begin{array}{c}10.4 \\
0\end{array}$ & $\mathrm{C}$ \\
\hline Sha Tin (13) & & & & & & & 1.7 & $\begin{array}{l}2.4 \\
1.3\end{array}$ & $\mathrm{D}$ & 2.3 & $\begin{array}{l}2.9 \\
1.7\end{array}$ & $\mathrm{D}$ \\
\hline Lai Chi Chong (16) & & & & & & & 2.8 & $\begin{array}{r}19.9 \\
0.4\end{array}$ & $\mathrm{D}$ & 1.4 & $\begin{array}{l}2.2 \\
0.6\end{array}$ & E \\
\hline
\end{tabular}

the results of Ireland \& Wootton (1977) in comparisons of contaminant profiles in the gastropods Thais (now Nucella) lapillus and Littorina littorea from 9 sites around the coast of Wales, UK. The carnivore $T$. lapillus exhibited distinct contaminant profiles from those of the herbivore Littorina littorea. More recent data of several authors (e.g. see Bryan \& Gibbs 1983, Bryan et al. 1985, Langston 1986) have confirmed such differences and expanded the current understanding of the use of biomonitors in defining time-averaged bioavailable contaminant levels in coastal waters.

The present study extends these observations to comparisons of barnacles and the mussel Perna viridis, employing an area with a well-defined pollution gradient. Although all 4 species studied are filter-feeders, the precise dietary preferences differ between the species. In particular, the barnacles Capitulum mitella and Tetraclita squamosa prefer larger zooplankton food, while Balanus amphitrite amphitrite is more microphagous in nature (Anderson 1981), capable of feeding on phytoplankton and detritus of a size range similar to that taken by $P$. viridis. The bioavailability of trace metals to each species may therefore differ, particularly where pollution gradients are subtle rather than extreme.

In general, the agreement between contamination profiles derived from data on mussels and barnacles was found to be good. Thus, for chromium, copper, lead and zinc, a high degree of correlation of site ranking (from most contaminated to least) was found, despite the fact that Perna viridis partially regulates its soft tissue levels of zinc. This partial regulation was first 
Table 6. Concentrations of zinc ( $\mu \mathrm{g} \mathrm{g}^{-1} \mathrm{dry}$ wt) and respective confidence limits (CL) in barnacles and mussels from Hong Kong waters, ranked in approximate order of decreasing contamination of samples. Letters in ANCOVA and ANOVA columns denote presence or absence of significant differences between samples

\begin{tabular}{|c|c|c|c|c|c|c|c|c|c|c|c|c|}
\hline \multirow[t]{2}{*}{ Location (code) } & \multicolumn{3}{|c|}{ Capitulum mitella } & \multicolumn{3}{|c|}{ Tetraclita squamosa } & \multicolumn{3}{|c|}{$\begin{array}{c}\text { Balanus amphitrite } \\
\text { amphitrite }\end{array}$} & \multicolumn{3}{|c|}{ Perna viridis } \\
\hline & $(\mathrm{Zn})$ & $\mathrm{CL}$ & ANCOVA & $(\mathrm{Zn})$ & $C I$ & ANCOVA & $(\mathrm{Zn})$ & $\mathrm{CL}$ & ANCOVA & $(\mathrm{Zn})$ & $\mathrm{CL}$ & ANOVA \\
\hline Hang Hau (12) & & & & & & & 11990 & $\begin{array}{l}14070 \\
10220\end{array}$ & A & 111 & $\begin{array}{r}147 \\
75\end{array}$ & $\mathrm{~A}$ \\
\hline Rennies Mill (11) & & & & & & & 11820 & $\begin{array}{r}14640 \\
9547\end{array}$ & $\mathrm{~B}$ & 109 & $\begin{array}{r}153 \\
65\end{array}$ & A \\
\hline Kowloon Pier (6) & & & & 7868 & $\begin{array}{l}9160 \\
6759\end{array}$ & A & & & & 108 & $\begin{array}{r}177 \\
39\end{array}$ & A \\
\hline Hung Hom (7) & 19890 & $\begin{array}{l}32120 \\
12320\end{array}$ & A & 6963 & $\begin{array}{r}11370 \\
4266\end{array}$ & B & & & & 118 & $\begin{array}{r}168 \\
68\end{array}$ & A \\
\hline Causeway Bay (8) & 9305 & $\begin{array}{r}14720 \\
5879\end{array}$ & $\mathrm{~B}$ & 3123 & $\begin{array}{l}5020 \\
2129\end{array}$ & B & & & & 150 & $\begin{array}{r}286 \\
14\end{array}$ & A \\
\hline Stonecutters (17) & 6374 & $\begin{array}{r}10640 \\
3819\end{array}$ & B & & & & & & & & & \\
\hline Queens Pier (5) & 4170 & $\begin{array}{l}7954 \\
2186\end{array}$ & C & 4086 & $\begin{array}{l}6261 \\
2666\end{array}$ & B & & & & 141 & $\begin{array}{r}208 \\
74\end{array}$ & A \\
\hline Reef Island (2) & 4471 & $\begin{array}{l}7586 \\
2635\end{array}$ & C & 4207 & $\begin{array}{l}6677 \\
2651\end{array}$ & B & & & & 114 & $\begin{array}{r}161 \\
67\end{array}$ & A \\
\hline Chai Wan Kok (3) & & & & & & & 9353 & $\begin{array}{r}11800 \\
7411\end{array}$ & C & 153 & $\begin{array}{r}247 \\
59\end{array}$ & A \\
\hline Kennedy Town (4) & & & & & & & & & & 146 & $\begin{array}{r}288 \\
4\end{array}$ & A \\
\hline North Point (9) & & & & 2534 & $\begin{array}{l}3083 \\
2084\end{array}$ & C & 7870 & $\begin{array}{r}10210 \\
6070\end{array}$ & C & 96 & $\begin{array}{r}154 \\
38\end{array}$ & B \\
\hline Kwun Tong (10) & & & & & & & 7276 & $\begin{array}{r}10050 \\
5269\end{array}$ & C & 115 & $\begin{array}{r}151 \\
79\end{array}$ & A \\
\hline Tung Chung (1) & & & & 2245 & $\begin{array}{l}3555 \\
1414\end{array}$ & C & 6491 & $\begin{array}{r}41600 \\
102\end{array}$ & $\mathrm{D}$ & 86 & $\begin{array}{r}103 \\
70\end{array}$ & B \\
\hline Wu Kwai Sha (15) & & & & & & & 4671 & $\begin{array}{l}5201 \\
4195\end{array}$ & $\mathrm{D}$ & 65 & $\begin{array}{l}85 \\
46\end{array}$ & $\mathrm{C}$ \\
\hline Tai Po Kau (14) & & & & & & & 4381 & $\begin{array}{l}5620 \\
3415\end{array}$ & E & 61 & $\begin{array}{l}80 \\
42\end{array}$ & C \\
\hline Sha Tin (13) & & & & & & & 3214 & $\begin{array}{l}3578 \\
2887\end{array}$ & F & 66 & $\begin{array}{l}94 \\
38\end{array}$ & C \\
\hline Lai Chi Chong (16) & & & & & & & 2726 & $\begin{array}{r}7688 \\
967\end{array}$ & $\mathrm{~F}$ & 53 & $\begin{array}{l}67 \\
39\end{array}$ & $\mathrm{D}$ \\
\hline Cape D'Aguilar (18) & 2852 & $\begin{array}{l}3944 \\
2062\end{array}$ & $\mathrm{D}$ & & & & & & & & & \\
\hline
\end{tabular}

proposed by Phillips (1985) on the basis of comparisons between zinc contamination profiles for $P$. viridis and other biomonitors in the Victoria Harbour area. Chan (1987) has recently confirmed the ability of $P$. viridis to partially regulate zinc, by the use of laboratory dosing techniques. Other bivalve species known to possess this ability include the mytilid Septifer virgatus (Phillips \& Yim 1981) and the hairy mussel Trichomya hirsuta (Klumpp \& Burdon-Jones 1982). Perna canaliculus also partially regulates zinc (V. Anderlini, pers. comm. to DJHP). Such regulatory abilities in bivalves do not appear to be as advanced as those in decapod crustaceans (Bryan 1968, 1976, White \& Rainbow 1982, 1984, Rainbow 1985, Bryan et al. 1986), but would nevertheless be an interesting area for further study.
The agreement between contamination profiles for elements other than cadmium in the barnacles and mussel studied here implies that the relative bioavailabilities of these metals at each site are similar with respect to all 4 biomonitoring species. However, profiles for cadmium differed substantially between the 3 barnacle species and the mussel, indicating the existence of subtle differences in bioavailability of this element from site to site, to species of divergent feeding habits in Hong Kong waters. The fact that cadmium shows no strong contamination gradient in the Hong Kong coastal environment (Phillips 1979a, 1985) is probably significant, in that such subtle site-to-site variations are more likely where severe contamination does not occur. For elements other than cadmium, the present data also agree well with previous studies of 

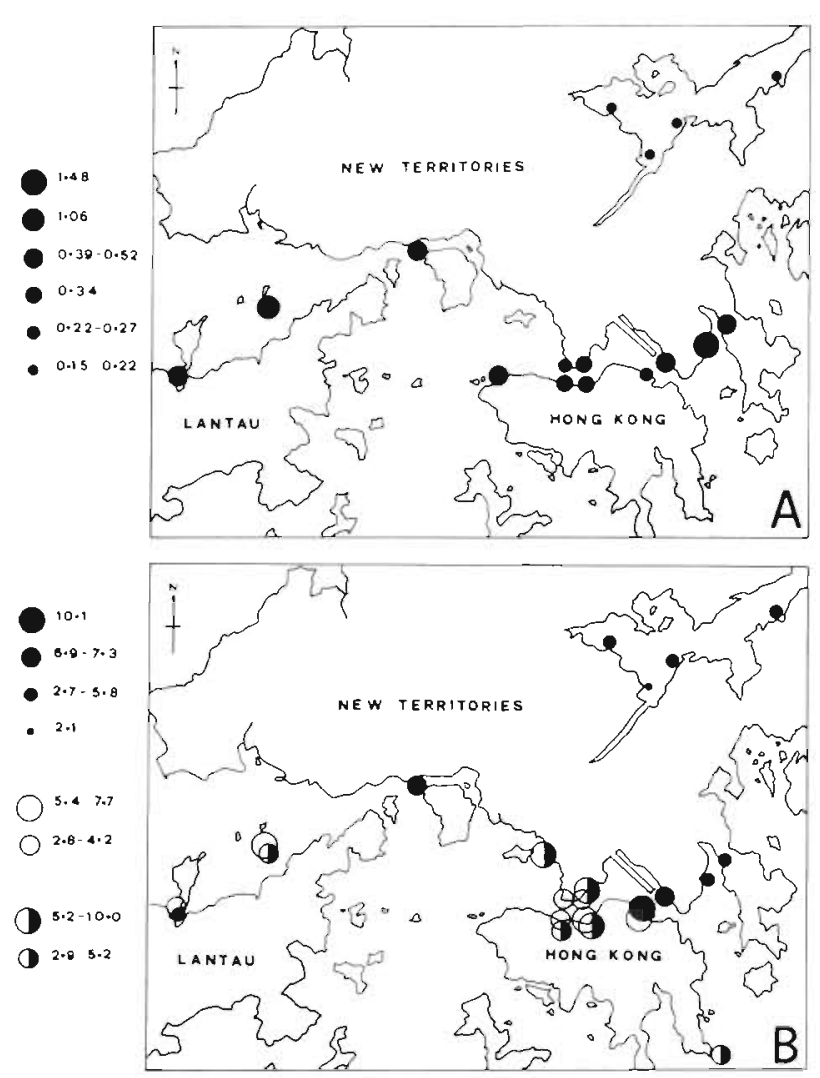

Fig. 2. Concentrations of cadmium ( $\mu \mathrm{g} \mathrm{g}^{-1}$ dry $w \mathrm{t}$ ) in barnacles and mussels from 18 sites in Hong Kong coastal waters. Data for barnacles refer to estimated concentrations for samples of standardized body weight, predicted by ANCOVA treatment; see text for details. Data for mussels shown as mean concentrations of samples, as analyzed. Sizes of circles denote significant differences between sites. (A.) Cadmium in Perna viridis. (B) Cadmium in Balanus amphritrite amphitrite (filled circles), Tetraclita squamosa (open circles), and Capitulum mitella (half-filled circles)

oysters, mussels (Septifer virgatus and $P$. viridis) and sediments (Phillips 1979a, 1985, Phillips \& Yim 1981) in this area of study.

The varying ranges in concentration of the 5 elements studied in barnacles and mussels are also of interest, as they reflect differences in the physiological handling of the metals by each species. The partial regulation of zinc by Perna viridis is an extreme case, although the overall contamination profile produced by studies of zinc in mussels nevertheless matched the profiles shown by barnacles, which exhibited very wide ranges in concentration of this element (Table 6). Data for zinc in these barnacles are reminiscent of results for oysters (Saccostrea glomerata) from these waters (Phillips 1979a); oysters, like barnacles, accumulate zinc to very high absolute concentrations.

The comparison of species with respect to copper and lead accumulation is also instructive. Perna viridis is unusual among mytilids, in that it accumulates very
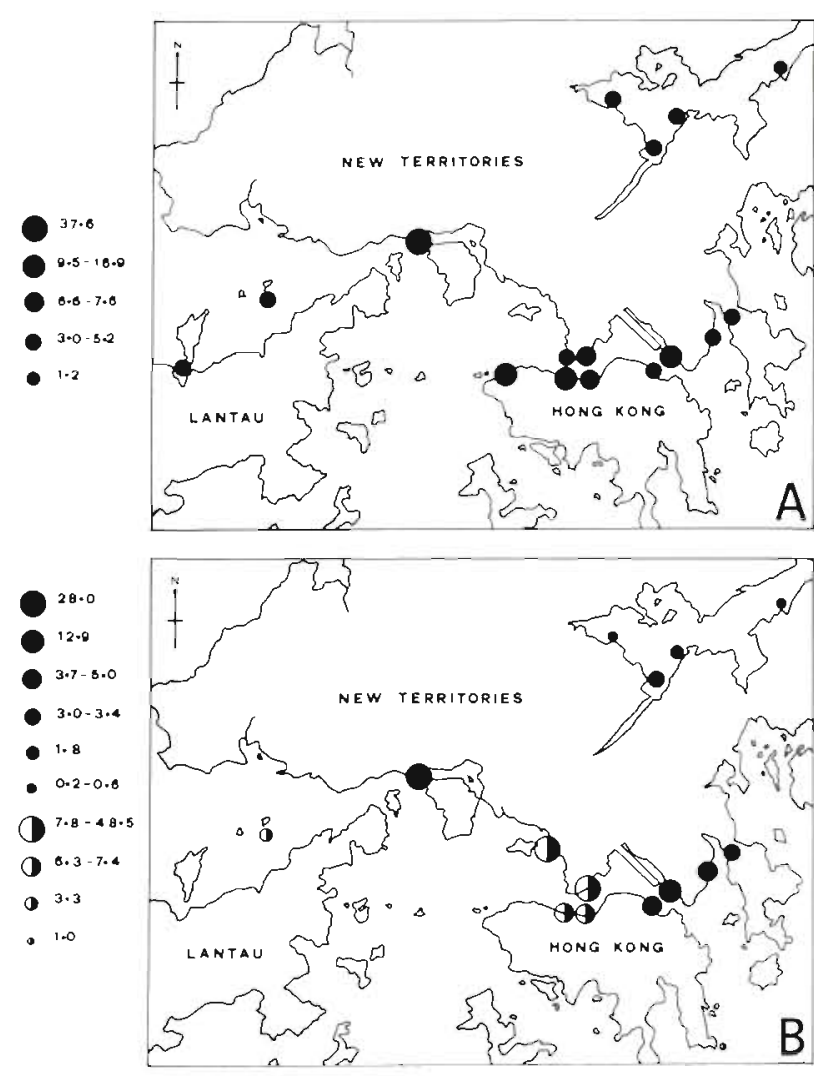

Fig. 3. Concentrations of chromium ( $\left.\mu \mathrm{g} \mathrm{g}^{-1} \mathrm{dry} w \mathrm{w}\right)$ in barnacles and mussels from 18 sites in Hong Kong coastal waters. Details as in legend to Fig. 2. (A) Chromium in Perna viridis (B) Chromium in Balanus amphitrite amphitrite (filled circles) and Capitulum mitella (half-filled circles)

large amounts of both of these elements in contaminated situations. Nevertheless, the 23-fold range between lowest and highest mean concentrations of copper in $P$. viridis from the present study is considerably smaller than the 59-fold range noted for weightstandardized copper concentrations in Balanus amphitrite amphitrite (Table 4). By comparison, the ranges between lowest and highest concentrations for lead in these 2 species (Table 5) are very similar, as are the absolute lead concentrations present. Such similarities and differences no doubt relate to the specific methods by which biomonitors sequester trace metals, presumably rendering them non-toxic in the process. Species that are poor regulators of accumulated trace metals are undoubtedly the most effective biomonitors, as intersite differences in concentrations of elements are greater and may overcome more easily the inherent variability among populations at any one site, permitting a more exact ranking of locations with respect to contamination.

Finally, it is worth noting here that insufficient data are available on tropical and subtropical areas of the world in terms of aquatic contamination. This situation 


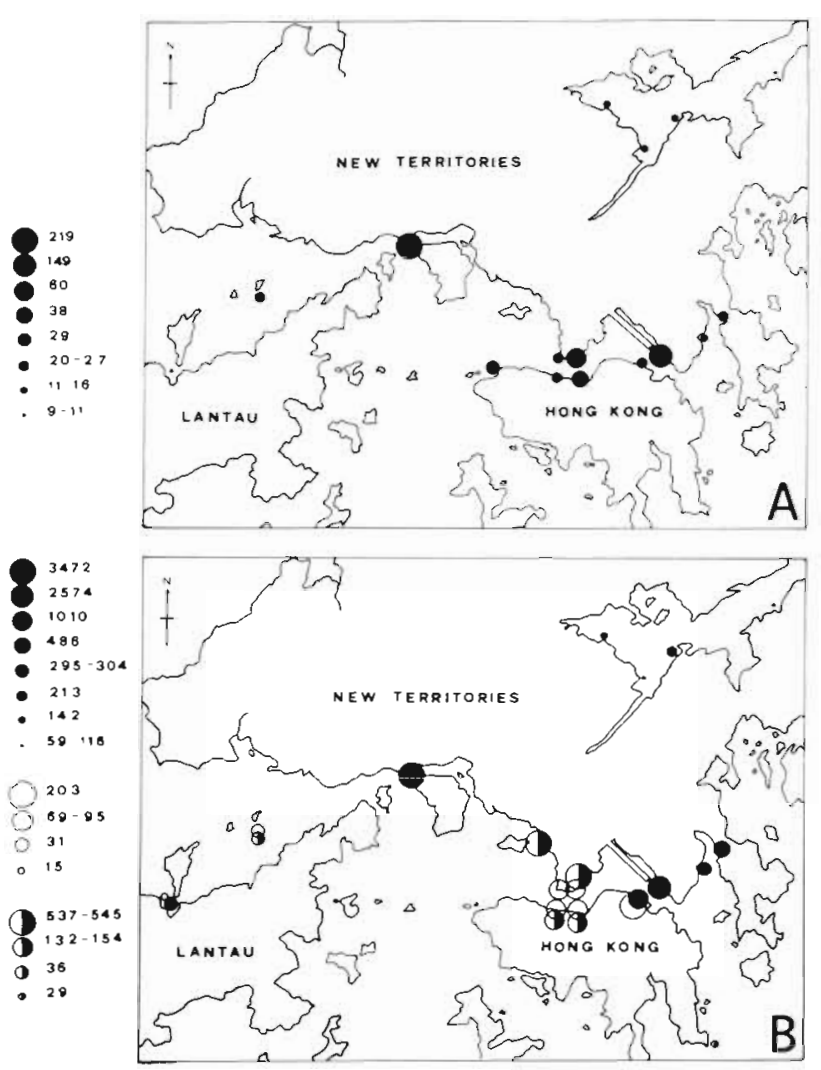

Fig. 4. Concentrations of copper $\left(\mu \mathrm{g} \mathrm{g}^{-1} \mathrm{dry} w \mathrm{w}\right)$ in barnacles and mussels from 18 sites in Hong Kong coastal waters. Details as in legend to Fig. 2. (A) Copper in Perna viridis. (B) Copper in Balanus amphitrite amphitrite (filled circles), Tetraclita squamosa (open circles), and Capitulum mitella (halffilled circles)

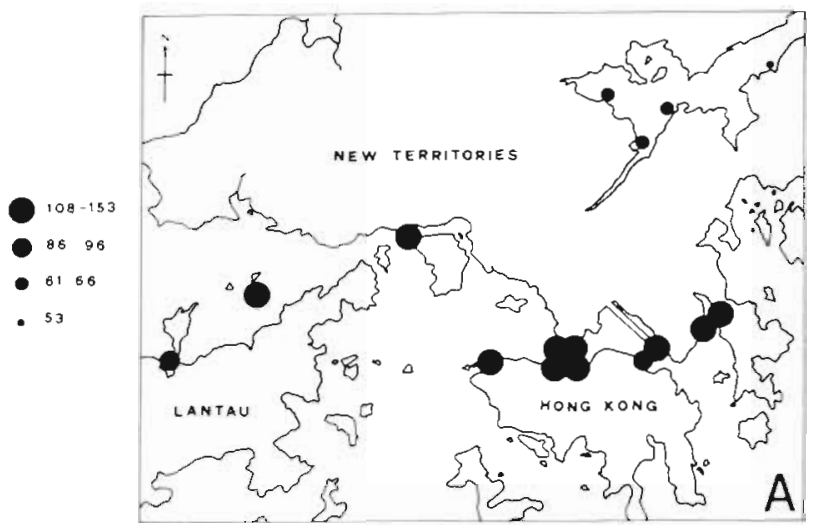

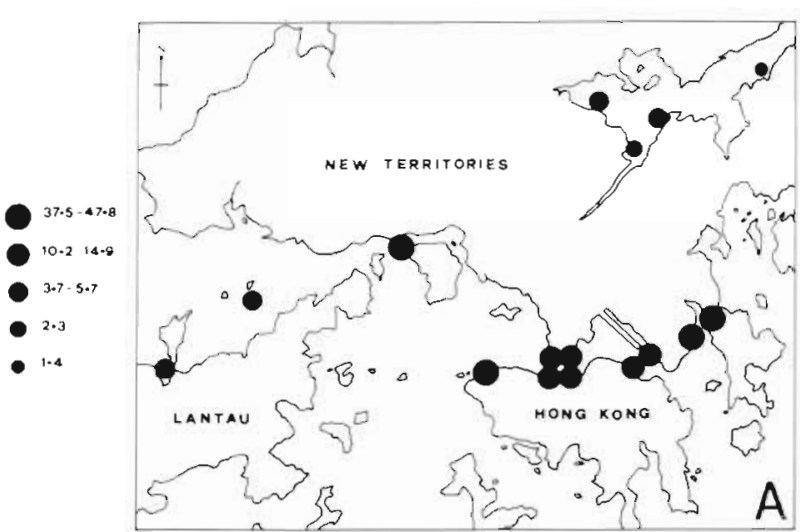

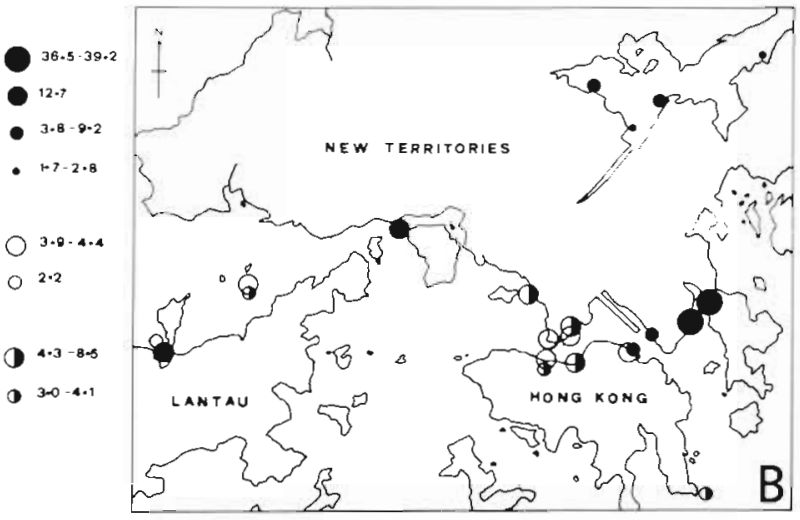

Fig. 5. Concentrations of lead ( $\mu \mathrm{g} \mathrm{g}^{-1} \mathrm{dry} \mathrm{wt}$ ) in barnacles and mussels from 18 sites in Hong Kong coastal waters. Details as in legend to Fig. 2. (A) Lead in Perna viridis. (B) Lead in Balanus amphitrite amphitrite (filled circles), Tetraclita squamosa (open circles), and Capitulum mitella (half-filled circles)

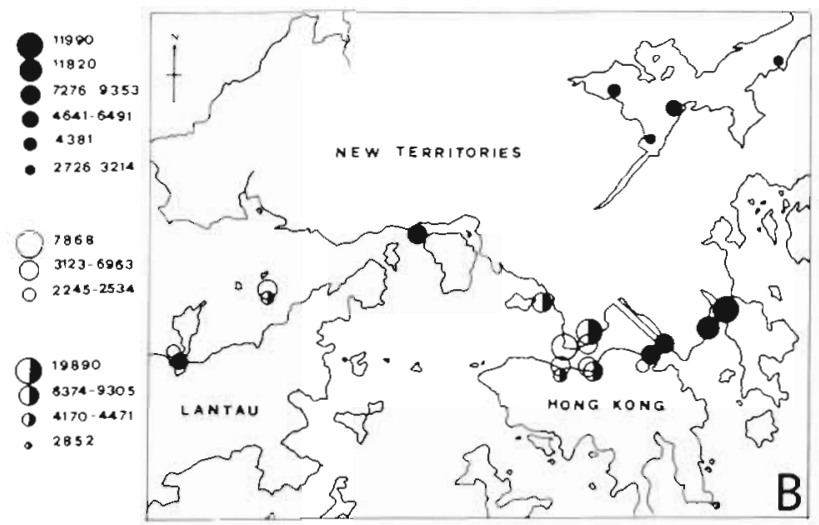

Fig. 6. Concentrations of zinc ( $\mu \mathrm{g} \mathrm{g}^{-1}$ dry wt) in barnacles and mussels from 18 sites in Hong Kong coastal waters. Details as in legend to Fig. 2. (A) Zinc in Perna viridis. (B) Zinc in Balanus amphitrite amphitrite (filled circles), Tetraclita squamosa (open circles), and Capitulum mitella (half-filled circles) 
continues despite the rapid urbanization and industrialization of many tropical nations. The use of the cosmopolitan biomonitoring species employed in the present study is recommended in such areas, as a means of both characterizing existing pollution levels and determining future changes in such levels.

Acknowledgements. PSR thanks Brian Morton and Graeme Green for assistance, and acknowledges financial support from the Royal Society, the Nuffield Foundation and E. A. Bevan of Queen Mary College. DJHP is grateful for the technical support of E. L. Chan and staff of EPD and the Government Laboratory in Hong Kong. Lee Phillips and Susan Prather helped in manuscript production.

\section{LITERATURE CITED}

Anderson, D. T. (1981). Cirral activity and feeding in the barnacle Balanus perforatus Bruguière (Balanidae), with comments on the evolution of feeding mechanisms in thoracican cirripedes. Phil. Trans. R. Soc. Lond., Ser. B 291: $441-449$

Bryan, G. W. (1968). Concentrations of zinc and copper in the tissues of decapod crustaceans. J. mar. biol. Ass. U. K. 48: 303-321

Bryan, G. W. (1976). Heavy metal contamination in the sea. In: Johnston, R. (ed.) Marine pollution. Academic Press, London, p. 185-302

Bryan, G. W., Gibbs, P. E. (1983). Heavy metals in the Fal Estuary, Cornwall: a study of long-term contamination by mining waste and its effects on estuarine organisms. Occ. Publ. mar. biol. Ass. U. K. 2: 1-112

Bryan, G. W., Hummerstone, L. G. (1977). Indicators of heavymetal contamination in the Looe Estuary (Cornwall) with particular regard to silver and lead. J. mar. biol. Ass. U. K. 57: 75-92

Bryan, G. W., Langston, W. J., Hummerstone, L. G. (1980). The use of biological indicators of heavy metal contamination in estuaries. Occ. Publ. mar. biol. Ass. U. K. 1: 1-73

Bryan, G. W., Langston, W. J., Hummerstone, L. G., Burt, G. R. (1985). A guide to the assessment of heavy metal contamination in estuaries using biological indicators. Occ. Publ. mar. biol. Ass. U. K. 4: 1-92

Bryan, G. W., Hummerstone, L. G., Ward, E. (1986). Zinc regulation in the lobster Homarus gammarus: importance of different pathways of absorption and excretion. J. mar. biol. Ass. U. K. 66: 175-199

Chan, H. M. (1987). An ecotoxicological study of trace metals in the green-lipped mussel Perna viridis (L) (Bivalvia: Mytilacea). Master of Philosophy thesis, University of Hong Kong

Goldberg, E. D., Bowen, V. T., Farrington, J. W., Harvey, G., Martin, J. H., Parker, P. L., Risebrough, R. W., Robertson,
W., Schneider, E., Gamble, E. (1978). The mussel watch. Environ. Conserv. 5: 101-125

Ireland, M. P., Wootton, R. J. (1977). Distribution of lead, zinc, copper and manganese in the marine gastropods, Thais lapillus and Littorina littorea, around the coast of Wales. Environ. Pollut. 12: 27-41

Klumpp, D. W., Burdon-Jones, C. (1982). Investigations of the potential of bivalve molluscs as indicators of heavy metal levels in tropical marine waters. Aust. J. mar. Freshwat. Res. 33: 285-300

Langston, W J. (1986). Metals in sediments and benthic organisms in the Mersey Estuary. Estuar. coast. Shel: Sci. 23: 239-261

Latouche, Y D., Mix, M. C. (1982). The effects of depuration, size and sex on trace metal levels in bay mussels. Mar. Pollut. Bull. 13: 27-29

NAS (1980). The International Mussel Watch. National Academy of Sciences, Washington, D. C.

Phillips, D. J. H. (1977). The use of biological indicator organisms to monitor trace metal pollution in marine and estuarine environments - a review. Environ. Pollut. 13; 281-317

Phillips, D. J. H. (1979a). The rock oyster Saccostrea glomerata as an indicator of trace metals in Hong Kong. Mar. Biol. 53: $353-360$

Phillips, D. J. H. (1979b). Trace metals in the common mussel, Mytilus edulis (L.), and the alga Fucus vesiculosus (L.) from the region of the Sound (Öresund). Environ. Pollut. 18: 13-43

Phillips, D. J. H. (1980). Quantitative aquatic biological indicators: their use to monitor trace metal and organochlorine pollution. Applied Science Publishers Ltd., London

Phillips, D. J. H. (1985). Organochlorines and trace metals in green-lipped mussels Perna viridis from Hong Kong waters: a test of indicator ability. Mar. Ecol. Prog. Ser. 21: $251-258$

Phillips, D. J. H., Yim, W W.-S. (1981). A comparative evaluation of oysters, mussels and sediments as indicators of :race metals in Hong Kong waters. Mar. Ecol. Prog. Ser. 6: 285-293

Rainbow, P. S. (1985). Accumulation of $\mathrm{Zn}, \mathrm{Cu}$ and $\mathrm{Cd}$ by crabs and barnacles. Estuar. coast. Shelf Sci. 21: 669-686

Rainbow, P. S. (1987). Heavy metals in barnacles. In: Southward, A. J. (ed.) Barnacle biology. A. A. Balkema, Rotterdam, p. 405-417

White, S. L., Rainbow, P. S. (1982). Regulation and accumulation of copper, zinc and cadmium by the shrimp Palaemon elegans. Mar. Ecol. Prog. Ser. 2: 143-152

White, S. L., Rainbow, P. S. (1984). Regulation of zinc corcentration by Palaemon elegans (Crustacea: Decapoda): zinc flux and effects of temperature, zinc concentration and moulting. Mar. Ecol. Prog. Ser. 16: 135-147

Wu, R. S. S. (1973). The distribution of littoral barnacles in Hong Kong. In: Morton, B. S. (ed.) Proceedings of Pacific Science Association Special Symposium in Marine Science. Hong Kong Government Printer, Hong Kong, p. $164-182$

This article was presented by Dr G. W. Bryan; it was accepted for printing on August 3, 1988 